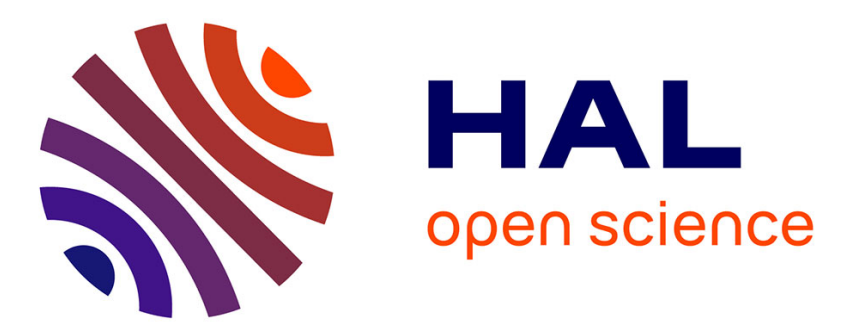

\title{
La Manche, nouvelle nécropole des migrants et de nos idéaux européens, mais qui sont les fossoyeurs?
}

\author{
Vincent Geisser
}

\section{To cite this version:}

Vincent Geisser. La Manche, nouvelle nécropole des migrants et de nos idéaux européens, mais qui sont les fossoyeurs?. Migrations Société, 2021, 4 (186), pp.3-10. 10.3917/migra.186.0003 . halshs03503292

\section{HAL Id: halshs-03503292 \\ https://shs.hal.science/halshs-03503292}

Submitted on 27 Dec 2021

HAL is a multi-disciplinary open access archive for the deposit and dissemination of scientific research documents, whether they are published or not. The documents may come from teaching and research institutions in France or abroad, or from public or private research centers.
L'archive ouverte pluridisciplinaire HAL, est destinée au dépôt et à la diffusion de documents scientifiques de niveau recherche, publiés ou non, émanant des établissements d'enseignement et de recherche français ou étrangers, des laboratoires publics ou privés. 


\title{
LA MANCHE, NOUVELLE NÉCROPOLE DES MIGRANTS ET DE NOS IDÉAUX EUROPÉENS, MAIS QUI SONT LES FOSSOYEURS?
}

\author{
Vincent Geisser
}

Centre d'Information et d'Etudes sur les Migrations Internationales | "Migrations Société »

$2021 / 4 \mathrm{~N}^{\circ} 186$ | pages 3 à 10

ISSN 0995-7367

DOI 10.3917/migra.186.0003

Article disponible en ligne à l'adresse :

https://www.cairn.info/revue-migrations-societe-2021-4-page-3.htm

Distribution électronique Cairn.info pour Centre d'Information et d'Etudes sur les Migrations Internationales.

(C) Centre d'Information et d'Etudes sur les Migrations Internationales. Tous droits réservés pour tous pays.

La reproduction ou représentation de cet article, notamment par photocopie, n'est autorisée que dans les limites des conditions générales d'utilisation du site ou, le cas échéant, des conditions générales de la licence souscrite par votre établissement. Toute autre reproduction ou représentation, en tout ou partie, sous quelque forme et de quelque manière que ce soit, est interdite sauf accord préalable et écrit de l'éditeur, en dehors des cas prévus par la législation en vigueur en France. Il est précisé que son stockage dans une base de données est également interdit. 


\section{La Manche, nouvelle nécropole des migrants et de nos idéaux européens, mais qui sont les fossoyeurs?}

Vincent GEISSER

Interrogé le 24 novembre 202I par l'Agence France Presse, quelques heures à peine après le décès de 27 migrants dans le naufrage de leur embarcation au large de Calais, le coordinateur d'une association de solidarité déclare: «Les gens meurent dans la Manche qui est en train de se transformer en cimetière à ciel ouvert, comme la Méditerranée $»^{I}$. Certes, on peut voir ici l'expression d'une "colère militante $»^{2}$ face à des drames humains qui ne cessent de se multiplier ces dernières années et qui touchent des migrants de toutes nationalités et de toutes conditions sociales, y compris des enfants et des adolescents. Mais ce sentiment de rage et d'impuissance est aussi très largement partagé par les universitaires, les experts et les responsables d'organisations humanitaires agissant dans le domaine des migrations internationales. En effet, leurs analyses s'apparentent de plus en plus à une forme de nécrologie migratoire, rendant compte des décès, des disparitions et des nombreuses victimes des violences perpétrées par la police, les milices ou les maffias. Écrire en ce début de $\mathrm{XXI}^{\mathrm{e}}$ siècle sur le fait migratoire, c'est quelque part accepter de devenir malgré soi un «nécrologue » de la migration. Toutefois, à la différence des nécrologies publiées dans la presse, il est quasiment impossible de restituer précisément la biographie du migrant défunt, condamné souvent à «l'anonymat éternel », à moins de céder à une forme de voyeurisme mortifère et malsain, à l'instar du portrait médiatique de Maryam Nouri, femme kurde de 24 ans, originaire du nord de l'Irak et décédée dans le naufrage au large de Calais. La publicisation de son histoire intime s'est déroulée sur un registre globalement exotique (femme, jeune, kurde, au visage de princesse orientale, etc.), occultant en partie les vraies raisons du drame: «Maryam Nouri Mohammad Amin rêvait d'une nouvelle vie. Cette jeune étudiante avait quitté le nord de l'Irak pour rejoindre son fiancé résidant au Royaume-Uni. Les deux amants, séparés, avaient fait la promesse de

1. Extrait de l'interview de Pierre Roques, coordinateur de L'Auberge des migrants (association loi 1901), cité dans : Le Monde/AGENCE FRANCE PRESSE, "Calais : au moins vingt-sept morts dans le naufrage d'une embarcation", le 24 novembre 2021, https://www.lemonde. $\mathrm{fr}$ /international/article/2021/11/24/calais-au-moins-vingt-migrants-morts-dans-le-naufra ge-d-une-embarcation-dans-la-manche_6103452_3210.html.

2. MATHIEU, Lilian, "Les ressorts sociaux de l'indignation militante. L'engagement au sein d'un collectif départemental du Réseau éducation sans frontière", Sociologie, vol. 1, $n^{\circ} 3,2010$, pp. 303-318. 
se retrouver $»^{3}$. Dans un tel récit, la part de responsabilité des autorités politiques dans le décès de la jeune femme n'est que rarement énoncée.

La dépolitisation de la représentation des drames migratoires - celui de Calais est un exemple parmi d'autres - constitue paradoxalement le fruit d'un véritable travail politique, jouant sur le registre des émotions et sur la rhétorique compassionnelle, qui s'apparente à une forme "d'humanisme de façade », tel le discours du président de la République, Emmanuel Macron, au lendemain du naufrage du 24 novembre: « Nous sommes tous émus par cette tragédie qui heurte chacun dans son intimité et dans ses valeurs [...]. C'est l'Europe dans ce qu'elle porte de plus profond - l'humanisme, le respect de la dignité de chacun - qui est endeuillée »" Cette lecture "dépolitisante» des tragédies migratoires est intrinsèquement liée à la désignation de boucs émissaires «faciles» (aisément méprisables par l'opinion publique), se situant généralement en dehors du champ politique et des sphères gouvernementales (les passeurs, les réseaux criminels, les maffias internationales, etc.), et appelant des réponses exclusivement sécuritaires. Dans ce registre, le président de la République a ainsi déclaré que « la France ne laissera[it] pas la Manche devenir un cimetière », demandant par ailleurs «le renforcement immédiat de Frontex $»^{5}$ et promettant de $«$ retrouver et condamner les responsables $»^{6}$. En 2015, la juriste Danièle Lochak avait déjà pointé l'hypocrisie et les limites d'une politique du «tout sécuritaire » dans la gestion des frontières maritimes, qui, non seulement ne résout pas le problème des décès, des blessés et des disparitions durant les traversées, mais contribue aussi à maintenir les migrants sous le joug de groupes criminels organisés : « Je pense par exemple à ce discours qui consiste à dire qu'il faut mettre fin à l'activité des passeurs et que tout rentrera dans l'ordre. C'est l'affirmation la plus stupide et la plus hypocrite qui soit. D'abord, s'il y a des passeurs, c'est que des personnes ont absolument besoin de recourir à leurs services à cause de la fermeture de toutes les routes légales et sûres pour venir en Europe. Ensuite, les moyens évoqués pour éradiquer leur

3. MAKOOI, Bahar, "Maryam, 24 ans, première victime identifiée du naufrage de migrants dans la Manche", France 24, le 28 novembre 2021, www.france24.com/fr/france/20211128maryam-24-ans-première-victime-identifiée-du-naufrage-de-migrants-dans-la-manche.

4. Le Monde/AGENCE FRANCE PRESSE, "Naufrage de migrants: Emmanuel Macron affirme que 'la France ne laissera pas la Manche devenir un cimetière'", 20 Minutes, le 25 novembre 2021, https://www.20minutes.fr/societe/3181179-20211124-naufrage-migrantsemmanuel-macron-affirme-france-laissera-manche-devenir-cimetiere.

5. L'Agence européenne de gardes-frontières et de gardes-côtes, établie par le règlement (UE) 2016/1624 du 14 septembre 2016 a remplacé I'Agence européenne pour la gestion de la coopération opérationnelle aux frontières extérieures des États membres de l'UE, qui avait été créée en 2004. Elle dispose de la même personnalité juridique et est connue sous le même nom : Frontex. Voir : RODIER, Claire, "Frontex, l'agence tout risque", Plein droit, vol. 87, $n^{\circ} 4$, décembre 2010, pp. 8-11.

6. France Info/AGENCE FRANCE PRESSE, "Naufrage de migrants : 'la France ne laissera pas la Manche devenir un cimetière', affirme Emmanuel Macron", le 24 novembre 2021, www.francetvinfo.fr/france/hauts-de-france/migrants-a-calais/naufrage-de-migrants-lafrance-ne-laissera-pas-la-manche-devenir-un-cimetiere-affirme-emmanuelmacron_4857765.html. 
activité supposent de livrer une véritable guerre, d'utiliser les armes de la guerre. Enfin, à supposer que l'on stoppe leur activité, cela signifie que les gens qui ont recours à ces passeurs ne pourront plus sortir de là où ils sont. lls seront torturés, tués et enfermés sur place au lieu de mourir en Méditerranée, puisque aujourd'hui c'est le choix qui leur est laissé $»^{7}$.

Bien sûr, il ne s'agit pas de nier l'existence de ces « réseaux de la honte » qui prospèrent sur le désespoir des migrants et les mettent en danger de mort, en les exposant à des conditions de traversée périlleuses, entassés sur des embarcations semi-rigides et sans protection particulière ${ }^{8}$. De ce point de vue, il convient de ne pas céder à une forme d'angélisme en occultant la nocivité des filières criminelles qui fonctionnent souvent sur des bases nationales, ethniques ou régionales, liées aux zones de départ. Les rares études sur le sujet ${ }^{9}$ témoignent du développement, au cours des cinq dernières années, de réseaux quasi professionnels de passeurs iraniens et irakiens qui agissent comme de véritables maffias à l'égard de leurs compatriotes ou des migrants d'autres nationalités, en leur extorquant de l'argent et en leur infligeant des pressions psychologiques et des violences physiques afin de les contraindre à embarquer dans des conditions inhumaines ${ }^{\mathrm{IO}}$. Mais, comme le soulignent aussi la plupart des rapports d'enquête, la tentation des migrants de recourir à des solutions extrêmes, s'explique en grande partie par l'épuisement des voies légales et les mesures de harcèlement pratiquées par les autorités des pays d'accueil et de transit qui les jettent dans les bras des réseaux criminels qui deviennent alors leur $«$ dernier recours ${ }^{\mathrm{II}}$.

En ce sens, les politiques migratoires des États européens marquées de plus en plus par des logiques sécuritaires, voire guerrières, encouragent les migrants à opérer des choix existentiels radicaux, quitte à mettre en danger leur intégrité physique et psychique. S’inspirant de l'approche initiée par Achille Mbembe, certains auteurs parlent ainsi de « nécro-politiques migratoires $»^{12}$, de « nécro-

7. BLARD, Mathieu, "Il faut supprimer le dispositif Dublin, mais il faut surtout supprimer Frontex" (entretien avec Danièle LOCHAK), Bondy Blog, le 10 juin 2015, http://bondyblog. liberation.fr/201506100001/daniele-lochak-il-faut-supprimer-le-dispositif-dublin-mais-ilfaut-surtout-supprimer-frontex.

8. TORONDEL, Loan, Exilés à la frontière franco-britannique : recherche sur les tentatives et les traversées de la Manche par voie maritime, 2018-2021, le 7 juin 2021, 142 p., https://associa tionsalam.org/Exiles-a-la-frontiere-franco-britannique-recherche-sur-les-tentatives-et-les.

9. SEBTAOUI, Nadia ; HARRISON, Christophe ; FOUILLEN, Servane, "Identification et protection des victimes de la traite dans un contexte de migration de transit", Les Cahiers du social, $\mathrm{n}^{\circ} 39,2017$, https://www.france-terreasile.org/images/stories/publications/pdf/Identification_et_protec tion_des_victimes_de_la_traite_dans_un_contexte_de_migration_de_transit.pdf.

10. TORONDEL, Loan, op. cit.

11. DÉFENSEUR DES DROITS, Exilés et droits fondamentaux, trois ans après le rapport Calais, Paris, décembre 2018, 76 p., www.defenseurdesdroits.fr/sites/default/files/atoms/files/rapport calais-num-14.12.18.pdf.

12. LEFÈVRE, Gabrielle, "Les 'nécro-politiques' migratoires: des crimes contre I'humanité", Entre les lignes, le 9 janvier 2018, www.entreleslignes.be/humeurs/zooms-curieux/lesnécro-politiques-migratoires-des-crimes-contre-l'humanité. 
pouvoir dans le contrôle migratoire $»^{13}$ ou encore du «pouvoir d'exposer à la mort $»^{I 4}$, c'est-à-dire la prérogative souveraine des États de décider de la vie et de la mort: «L'expression ultime de la souveraineté réside largement dans le pouvoir et la capacité de dire qui pourra vivre et qui doit mourir. Faire mourir ou laisser vivre constituent donc les limites de la souveraineté, ses principaux attributs. Être souverain c'est exercer son contrôle sur la mortalité et définir la vie comme le déploiement et la manifestation du pouvoir $»^{15}$. Si ces formulations peuvent paraître abruptes, elles ont au moins le mérite de mettre en exergue les logiques belliqueuses qui structurent aujourd'hui les politiques migratoires des États, démocratiques et/ou autoritaires, qui transforment les migrants en «suspects », « adversaires » ou « ennemis », en dépit de discours compassionnels de circonstance. La nécropolitique migratoire constitue une manifestation paroxystique de l'humanisme sécuritaire que nous évoquons au fil de nos éditoriaux ${ }^{16}$ : contrôler, surveiller et expulser les migrants soi-disant pour leur bien ou, du moins, pour celui de leur société d'origine.

À ce titre, la gestion de la frontière maritime dans la zone située entre Calais et Grande-Synthe constitue à la fois une illustration et un terrain d'expérimentation du processus de militarisation des politiques migratoires ${ }^{17}$. Comme le relèvent les auteurs d'un récent rapport parlementaire, « $85 \%$ des dépenses exécutées financent la sécurisation des territoires et $15 \%$ sont dédiées à la prise en charge sanitaire, sociale ou humanitaire des populations migrantes $»^{18}$. À l'instar des frappes dites «chirurgicales» qui sont tout aussi destructrices en vies humaines que les bombardements massifs, les autorités politiques prônent la construction d'une «frontière intelligente $»^{\mathrm{I} 9}$, euphémisme pour désigner l'appel aux technologies sécuritaires les plus sophistiquées. Cette politique de militarisation de la frontière maritime n'est cependant

13. SAHRAOUI, Nina, "Le 'nécropouvoir' dans le contrôle migratoire et ses implications genrées dans les périphéries postcoloniales françaises de l'Océan indien", Migrations société, vol. 32, n 182, octobre-décembre 2020, pp. 29-42.

14. RITAINE, Évelyne, "Du pouvoir d'exposer à la mort à/par la frontière", Cultures \& conflits, n 99-100, Automne-Hiver 2015, pp. 145-148.

15. MBEMBE, Achille, "Nécropolitique", Raisons politiques, vol. 21, n 1, 2006, pp. 29-60 (voir p. 29).

16. GEISSER, Vincent, "Leçons de l'élection européenne de 2019. L'humanisme sécuritaire et néolibéral du président Macron: antidote ou iatrogénie à la 'lèpre nationaliste'?", Migrations société, vol. 31, n 176, avril-juin 2019, pp. 3-15.

17. GILIBERTI, Luca, "La militarisation de la frontière franco-italienne et le réseau de solidarité avec les migrant.e·s dans la Vallée de la Roya", Mouvements, $\mathrm{n}^{\circ}$ 93, Printemps 2018 pp. 149-155.

18. ASSEMBLÉE NATIONALE, Rapport fait au nom de la Commission d'enquête sur les migrations, les déplacements de populations et les conditions de vie et d'accès au droit des migrants, réfugiés et apatrides en regard des engagements nationaux, européens et internationaux de la France, présenté par Sébastien NADOT (président) et Sonia KRIMI (rapporteuse), enregistré le 10 novembre 2021, 453 p. (voir p. 125).

19. BOITIAUX, Charlotte, "Des quads, des $4 \times 4$, des caméras... Dans le nord de la France, toujours plus de moyens pour lutter contre les traversées clandestines", InfoMigrants, le 23 novembre 2021, https://www.infomigrants.net/fr/post/36684/des-quads-des-4x4-des-cameras-dans-lenord-de-la-france-toujours-plus-de-moyens-pour-lutter-contre-les-traversees-clandestines. 
pas récente. Depuis plus de 30 ans, tous les traités franco-britanniques (Sangatte I99I, Le Touquet 2003, et Sandhurst 20I8) reposent sur une conception hyper-sécuritaire de la coopération bilatérale au détriment des aspects culturels, sociaux et sanitaires ${ }^{20}$. Cette orientation guerrière de la lutte contre les migrations dites « clandestines » a d'ailleurs été accentuée par le Plan d'action conjoint franco-britannique du 24 janvier 20I9, qui prévoit le déploiement de nouvelles technologies de surveillance afin de bâtir une "frontière modèle » pour les années à venir : " Des caméras de surveillance supplémentaires seront placées stratégiquement dans les ports français, ainsi que dans des zones où les migrants peuvent tenter d'embarquer. Celles-ci fourniront des images de vidéosurveillance au Centre conjoint d'information et de coordination de Calais qui réunit des personnels des forces de l'ordre françaises et britanniques. Les patrouilles maritimes et aériennes de surveillance de la Manche seront renforcées, et les forces de l'ordre françaises et britanniques partageront davantage d'informations relatives à ces exercices. Les patrouilles pédestres des plages et zones côtières par la police française seront augmentées, dans le but d'interrompre les tentatives de passage. Les gouvernements français et britannique ont souligné que tous les auteurs d'infractions seront poursuivis $»^{21}$. Sous couvert de protéger les individus face aux agissements des réseaux criminels, c'est en réalité une véritable guerre aux migrants qui est menée conjointement par la France et le Royaume-Uni, sans faire de distinction entre les différentes catégories: "Nous avertissons également les migrants sur le fait que nous utiliserons l'ensemble des possibilités juridiques qui s'offrent à nous dans la gestion du processus migratoire. Dans ce contexte, le retour de migrants du Royaume-Uni vers la France, ou de France vers le Royaume-Uni, sera reconduit très rapidement conformément à nos obligations internationales et aux législations nationales respectives. Ceci inclut notamment le recours au Règlement de Dublin et aux vols conjoints vers les pays d'origine $»^{22}$. Fidèle à cette conception militaire de la lutte contre les migrations dites «illégales », qui s'inspirent en partie des méthodes antiguérilla ${ }^{23}$, Londres et Paris ont créé, dans la foulée, une cellule de renseignement franco-britannique dans le but de "rassembler, centraliser et analyser » les informations opérationnelles, " afin d'empêcher les passages et de démanteler les gangs qui les organisent $»^{24}$. Sur cette question, le Brexit n'a rien changé. Il aurait même plutôt aggravé la situation de précarité et d'insécurité des migrants qui se retrouvent à la merci à la fois des groupes

20. TORONDEL, Loan, op. cit., (voir p. 34).

21. MINISTÈRE DE L'INTÉRIEUR, HOME DEPARTMENT, Communiqué de presse à propos du Plan d'action commun dans le domaine de la lutte contre l'immigration irrégulière impliquant de petites embarcations traversant La Manche, le 24 janvier 2019, https://mobile.interieur. gouv.fr/Archives/Archives-des-communiques-de-presse/2019-Communiques/Plan-daction-de-lutte-contre-I-activite-des-migrants-dans-la-Manche.

22. Ibidem.

23. BIGO, Didier, "L'immigration à la croisée des chemins sécuritaires", Revue européenne des migrations internationales, vol. 14, $\mathrm{n}^{\circ} 1,1998, \mathrm{pp} .25-46$.

24. TORONDEL, Loan, op. cit. (voir p. 41). 
maffieux, des sociétés de sécurité privées mais aussi des forces de l'ordre public : «Dans le cadre du Brexit, dont l'application est devenue effective le $\mathrm{I}^{\mathrm{er}}$ janvier 202I, ces mécanismes de coordination entre les deux pays tendent à se renforcer dans une logique toujours plus répressive. Depuis juillet 2020, et la rencontre à Calais entre Gérald Darmanin et Priti Patel, représentant respectivement le ministère de l'Intérieur français et le Home Office britannique, un durcissement réel des discours et annonces sur le thème migratoire est à noter de part et d'autre de la Manche ${ }^{25}$.

Toutefois, on aurait tort de croire qu'il s'agit d'une rhétorique sécuritaire visant simplement à effrayer les migrants et à intimider les acteurs de la solidarité qui leur viennent en aide. Sur le terrain, elle se traduit également par une stratégie de harcèlement quasi quotidienne des migrants qui s'est amplifiée ces dernières années, notamment depuis le démantèlement de la "jungle de Calais » en décembre $2016^{26}$, comme le notent les auteurs du rapport de l'organisation Human Rights Observers : «Les violences quotidiennes infligées par les forces de l'ordre visent à l'épuisement physique et moral des personnes bloquées à la frontière : coups, blessures, privation de sommeil et de nourriture, surveillance constante, contrôle et restriction des espaces de vie et humiliations. La précarité organisée de leurs conditions de vie et l'absence d'hébergement décent qui les pousse à devoir vivre dans des campements précaires démontrent la volonté institutionnelle de maintenir dans l'insécurité les personnes exilées ${ }^{27}$. Au-delà des opérations de maintien de l'ordre, qui apparaissent somme toute légales et légitimes dans un État démocratique comme la France, c'est en réalité une véritable politique de la terre brûlée qui est pratiquée par les pouvoirs publics et les autorités sécuritaires, allant jusquà modifier l'écosystème local pour mieux chasser les migrants: "Cette politique violente se base notamment sur l'expulsion forcée des personnes de leurs lieux de vie informels, mais aussi sur différentes mesures, qui visent à dissuader les personnes exilées de se rendre aux points de transit du littoral et [à] les tenir le plus loin possible de la frontière. Une des mesures phares de 2020 : le déboisement des lieux de vie, que ce soit à Calais ou à Grande-Synthe. Le déboisement fait partie des techniques utilisées depuis longtemps pour impliquer la nature, telle que façonnée par l'humain, dans la sécurisation de la frontière $»^{28}$.

La politique de répression anti-migrants est d'autant plus efficace qu'elle se fonde sur une stratégie d'invisibilisation de la violence, orchestrée par ses ordonnateurs afin qu’il n'y ait pas de témoin: «Le périmètre établi par les

25. HUMAN RIGHTS OBSERVERS, Observations des violences d'État à la frontière franco-britannique Calais et Grande-Synthe, Rapport annuel 2020, 49 p. (voir p. 4), http://www.laubergedesmigra nts.fr/wp-content/uploads/2021/05/Rapport-annuel-2020-Human-Rights-Observers-HRO.pdf.

26. AGIER, Michel; BOUAGGA, Yasmine; GALISSON, Maël, HANAPPE, Cyrille; PETTE, Mathilde WANNESSON, Philippe, La jungle de Calais, Paris : Presses universitaires de France, 2018, $224 \mathrm{p}$

27. HUMAN RIGHTS OBSERVERS, op. cit. (voir p. 34).

28. Ibidem (voir p. 4). 
forces de l'ordre au début de l'expulsion constitue surtout une entrave délibérée aux tentatives de documentation et participe à l'invisibilisation des violences perpétrées à la frontière contre les personnes qui s'y trouvent bloquées ${ }^{29}$. Les ONG humanitaires, les associations locales de solidarité (Salam, L'Auberge des migrants, Utopia 56, etc.), les travailleurs sociaux et les journalistes sont ainsi empêchés d'accéder aux lieux d'intervention durant les opérations d'expulsion, ils sont donc dans l'incapacité de faire leur travail d'assistance aux migrants et d'information auprès du grand public.

Enfin, conformément aux politiques néolibérales promues depuis plus de 30 ans par les différents gouvernements de gauche comme de droite ${ }^{30}$, et renforcées sous la présidence Macron, l'on observe le développement de logiques de privatisation de la surveillance et de la répression, un partenariat public-privé dirigé contre les migrants : « Les membres de sociétés de sécurité privées, en particulier aux ports, sont aussi à l'origine de nombreux actes de violences. Lâchés de chiens, passages à tabac, courses poursuites, les moyens employés par ces équipes de sécurité s'alignent sur les méthodes utilisées par les forces de l'ordre pour rendre la frontière aussi dangereuse que possible $»^{31}$.

On pouvait penser que le «drame de Calais » du 24 novembre contribuerait à infléchir partiellement la politique du «tout sécuritaire» conduite par le gouvernement, en l'incitant à adopter une approche nouvelle dans la lutte contre les « traversées illégales » dans la Manche. Or, non seulement le ministre de l'Intérieur, Gérald Darmanin, n’a pas esquissé la moindre autocritique des effets pervers de «sa» politique répressive mais il a aussi annoncé une série de mesures visant à consolider le processus de militarisation de la gestion de la frontière maritime ${ }^{32}$.

Cependant, cette politique de militarisation de la lutte contre l'immigration dite « clandestine » est loin de faire l'unanimité, y compris dans les milieux de la majorité présidentielle. Quelques jours avant le « drame de Calais », un rapport parlementaire coordonné par la députée de la quatrième circonscription de La Manche, Sonia Krimi (LREM), s'est attaché à déconstruire les orientations sécuritaires de la politique migratoire du gouvernement, en soulignant non seulement les atteintes aux droits fondamentaux mais aussi son inefficacité totale en termes de gestion des flux migratoires: "S'il n'est pas souhaitable de laisser se former une nouvelle "jungle", la simple poursuite de la politique de lutte contre les "points de fixation" n'est pas tenable. Il convient de mettre "fin à cette traque" dénoncée par la Défenseure des droits. L'argument avancé par les pouvoirs publics pour justifier cette

29. HUMAN RIGHTS OBSERVERS, op. cit. (voir p. 17).

30. MATH, Antoine, "Une politique au service du néo-libéralisme", Plein droit, n 51 , novembre 2001, pp. 27-29.

31. HUMAN RIGHTS OBSERVERS, op. cit., (voir p. 36).

32. AGENCE FRANCE PRESSE/Le Monde, "Mort de migrants dans la Manche: Gérald Darmanin critiqué, l'opposition dénonce une 'honte' et un 'drame de l'inhumanité'", le 25 novembre 2021, https://www.lemonde.fr/politique/article/2021/11/25/mort-de-27-migrants-dansla-manche-gerald-darmanin-critique-I-opposition-denonce-une-honte-et-un-drame-deI-inhumanite_6103553_823448.html. 
politique est celui du risque d'un prétendu "appel d'air". Pourtant, les dernières années démontrent que mêmes les conditions d'accueil les plus indignes et les conditions de passage les plus dangereuses ne dissuadent pas les personnes exilées de vouloir tenter leur chance pour l'Angleterre. En effet, force est de constater que l'extrême sécurisation de la frontière franco-britannique à l'œuvre n'a pas les effets attendus sur les flux migratoires ${ }^{33}$.

Le rapport prône ainsi une "désectorisation sécuritaire » de la politique migratoire qui ne serait plus confiée exclusivement au seul ministère de l'Intérieur mais s'inscrirait dans une logique interministérielle: "Après la Seconde Guerre mondiale, la politique d'immigration était partagée entre le ministère des Affaires étrangères, le ministère des Affaires sociales et le ministère de l'Intérieur. Ce partage a globalement bien fonctionné pendant quarante ans, chaque ministère ayant ainsi son approche de la question migratoire. Progressivement, les préoccupations d'ordre sécuritaire l'ayant emporté, le ministère de l'Intérieur a largement fait valoir son point de vue au détriment des autres administrations au nom de la recherche d'efficacité [...]. Or, la question migratoire, les conditions d'entrée sur le territoire mais aussi l'accueil et l'intégration des migrants nécessitent la mise en synergie [d']un nombre important de politiques publiques intérieures, mais aussi extérieures ${ }^{34}$.

L'intérêt du rapport parlementaire coordonné par Sonia Krimi n'est pas tant le caractère avant-gardiste des propositions et des recommandations qui reprennent, en grande partie, les observations et les diagnostics établis depuis plusieurs années par les universitaires, les acteurs de terrain et les responsables des associations de solidarité, que l'ouverture d'un débat au sein même des milieux gouvernementaux, des hauts fonctionnaires et de certains cercles proches du président de la République. Certes, la position de la députée de la Manche qui, dans un passé récent, s'est déjà illustrée par des déclarations critiques sur la gestion répressive du mouvement des Gilets jaunes ${ }^{35}$ et sur la loi contre le séparatisme ${ }^{36}$, apparaît relativement isolée dans le bloc majoritaire, mais elle a le mérite d'ouvrir une «brèche humaniste» dans une campagne présidentielle qui s'annonce focalisée sur les questions identitaires et sécuritaires, avec aux premières loges de la scène politico-électorale les fossoyeurs de nos idéaux démocratiques.

Marseille, le 8 décembre 2021

33. ASSEMBLÉE NATIONALE, op. cit. (voir p. 125).

34. Ibidem, (voir pp. 81-82).

35. BOUDET, Alexandre, "Sonia Krimi, députée LREM, met un gilet jaune pour les soutenir", Huffpost, le 23 novembre 2018, www.huffingtonpost.fr/2018/11/23/sonia-krimi-deputeeIrem-met-un-gilet-jaune-pour-les-soutenir_a_23598286/.

36. CLERC, Olivier, "Sonia Krimi : 'Mon vote pour la loi confortant les principes républicains n'est pas acquis'", Ouest France, le 9 décembre 2020, www.ouest-france.fr/normandie/ cherbourg-en-cotentin-50100/sonia-krimi-mon-vote-pour-la-loi-confortant-les-principe s-republicains-n-est-pas-acquis-7079188. 